\title{
Propuesta de caracterización del nivel de servicio por línea de atraque en contratos de concesión de terminales portuarias de contenedores
}

\author{
Arturo Monfort Mulinas \\ Director I+D+i, Fundación Valenciaport, España \\ José Aguilar Herrando
}

Catedrático de explotación de puertos, Universitat Politècnica de València, España

\section{RESUMEN}

Un elemento básico en la competitividad de los países y regiones es la componente logística (coste/tiempo) que se produce en los nodos y modos que conforman las correspondientes cadenas de suministro. Las terminales portuarias son nodos estratégicos en las cadenas logístico-portuarias. Con carácter universal la política de transportes ha apostado por el modelo de gobernanza portuaria landlord que se materializa en inversiones y explotación portuaria a través de asociaciones público-privadas, habitualmente bajo el instrumento de un contrato de concesión. En los contratos de concesión se recogen los derechos y obligaciones de las partes, a lo largo del plazo concesional que es dilatado, al objeto de que el operador privado pueda recuperar su inversión. De lo contemplado en el mismo resultará el nivel de servicio de la terminal en los subsistemas de línea de atraque (carga/descarga de buques), almacenamiento y recepción/entrega al transporte terrestre. Es imprescindible que los indicadores que se contemplen sean capaces de caracterizar de hecho los niveles de servicio ofertados. El nivel de servicio del subsistema de carga/descarga de buques o de línea de atraque debe incorporar por un lado la calidad de servicio ofertada en términos de esperas en relación con los tiempos de atraque; y por otro, la productividad en el atraque. Éste resultará del dimensionamiento de la terminal en longitud de línea de atraque y grúas de muelle para dar respuesta a la demanda de tráfico por vía marítima.

En la ponencia se propone la caracterización del nivel de servicio por línea de atraque para su inclusión tras la oportuna particularización en los contratos de concesión de terminales portuaria de contenedores.

\section{LA GOBERNANZA DE LOS PUERTOS: LAS CONCESIONES}

\subsection{El concepto de gobernanza portuaria}

El concepto de gobernanza portuaria desembarca en los puertos hace aproximadamente una década de la mano del mundo académico, acompañado del de "devolución" (“devolution", Brooks y Cullinane, 2006) en el sentido de delegación o transferencia de poderes, materializada a través de estrategias de descentralización, comercialización, corporatización y privatización que articulan en cada caso el correspondiente modelo de gestión portuario (Fig. 1). 


\begin{tabular}{|c|c|c|c|c|}
\cline { 2 - 5 } \multicolumn{2}{c|}{} & \multicolumn{2}{c|}{ Inversiones } & \multicolumn{2}{c|}{ Prestación servicios } \\
\hline Modelo & Infraestructura & Superestructura & Estiba & Otros \\
\hline Public service & Público & Público & Público & Mayoría Pública \\
\cline { 3 - 5 } Tool & Público & Público & Privada & Público/privada \\
\hline Landlordport & Público/privada & Privada & Privada & Público/privada \\
\hline Private service & Privada & Privada & Privada & Mayoría Privada \\
\hline
\end{tabular}

\section{Fig. 1 - Modelos de gestión portuaria}

En los puertos que responden al modelo landlord, la infraestructura ha venido siendo financiada por el Gobierno y la Autoridad Portuaria, y la superestructura y los equipos por el sector privado. En algunas ocasiones, la Autoridad Portuaria, obligada a realizar continuamente importantes inversiones en accesos, infraestructuras en zonas comunes e incluso en sistemas de comunicación e información, ha incorporado la intervención del sector privado también en la financiación de infraestructuras, teniendo en cuenta las limitaciones habituales de los recursos económicos disponibles. En los últimos años, en algunos casos, las empresas privadas han financiado los muelles y rellenos de la terminal portuaria objeto de concesión.

\subsection{Los contratos de concesión de terminales portuarias}

La herramienta más universal de "privatización portuaria" -entendida como el proceso o tendencia de mayor participación del sector privado en la inversión y prestación de servicios portuarios, sin que esto suponga, salvo en casos excepcionales (Reino Unido), la venta de los activos de los puertos por el sector público al privado- es el del instrumento del contrato de concesión (tipología Build-Operate-Transfer, BOT), personalizado en cada país de acuerdo con el correspondiente marco legal. Tal instrumento entra en la categoría de la más reciente denominación de Asociación Público Privada (APP).

En términos generales, desde la perspectiva del derecho administrativo, se puede definir el contrato de concesión portuaria de una terminal como aquel que otorga el sector público al privado para el uso del espacio portuario en el desarrollo de la prestación de servicios específicos. Dado que los objetivos y recursos que aportan los distintos agentes directamente relacionados con la explotación de la terminal, se producen conflictos de interés relacionados con la capacidad y nivel de servicio de la infraestructura y de las operaciones (Fig. 2). 


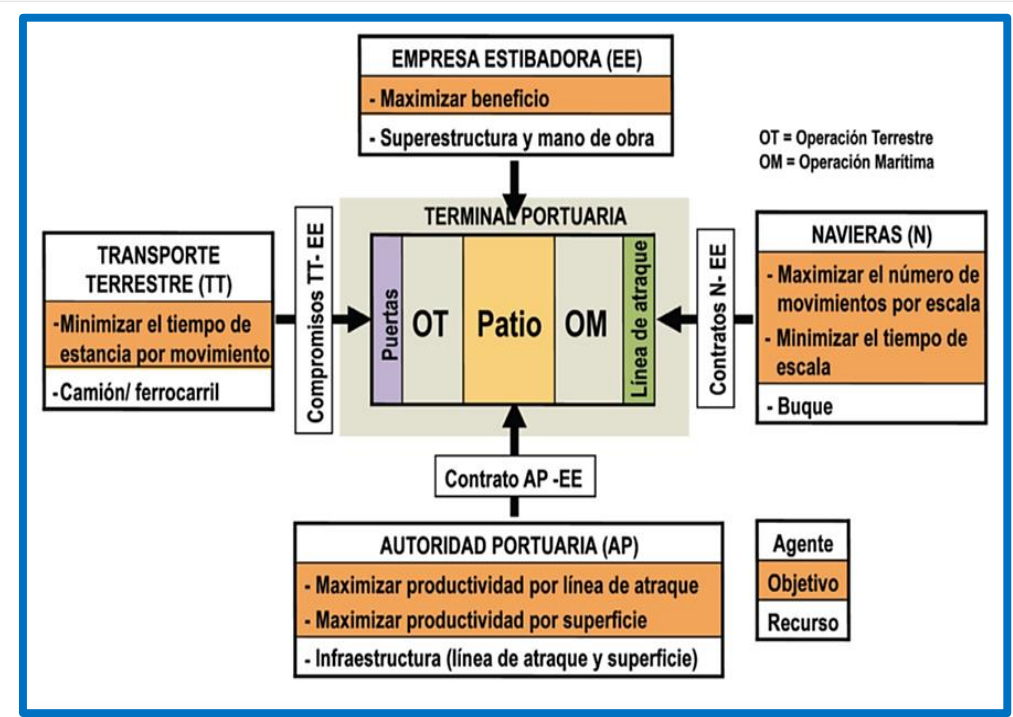

Fig. 2 - Contrato de concesión y conflicto de intereses

\section{LAS TERMINALES PORTUARIAS DE CONTENEDORES: CAPACIDAD}

\subsection{El concepto de terminal portuaria de contenedores}

Desde una perspectiva histórica puede afirmarse que el concepto de terminal portuaria es una concepción moderna de la explotación portuaria (modelo landlord), que con anterioridad hacía uso del concepto de muelle (Rodríguez, 1977). En este sentido, lo significativo es que una alineación de atraques o muelles, normalmente especializada, pasa a ser gestionada, por un único prestador de servicios a través de fórmulas de asociación público privadas. No obstante, por ejemplo en el caso español, ya desde el siglo XIX se empleó la figura de la concesión demanial, tanto para la construcción de un puerto como para su explotación; por ejemplo, el Puerto de Gandía en 1884 (Donnet, 1910).

Una terminal portuaria es un intercambiador modal que suele disponer de un área de almacenamiento en tierra para coordinar los diferentes ritmos de llegadas de la mercancía vía marítima y terrestre (Monfort et al., 2001). Es un sistema integrado, con conexión física y de información con las redes de transporte terrestres y marítimas (Monfort et al., 2011). Para su análisis se considera que está compuesto por cuatro subsistemas (Fig. 3): carga/descarga (línea de atraque), transporte horizontal o interconexión, almacenamiento y recepción/entrega.

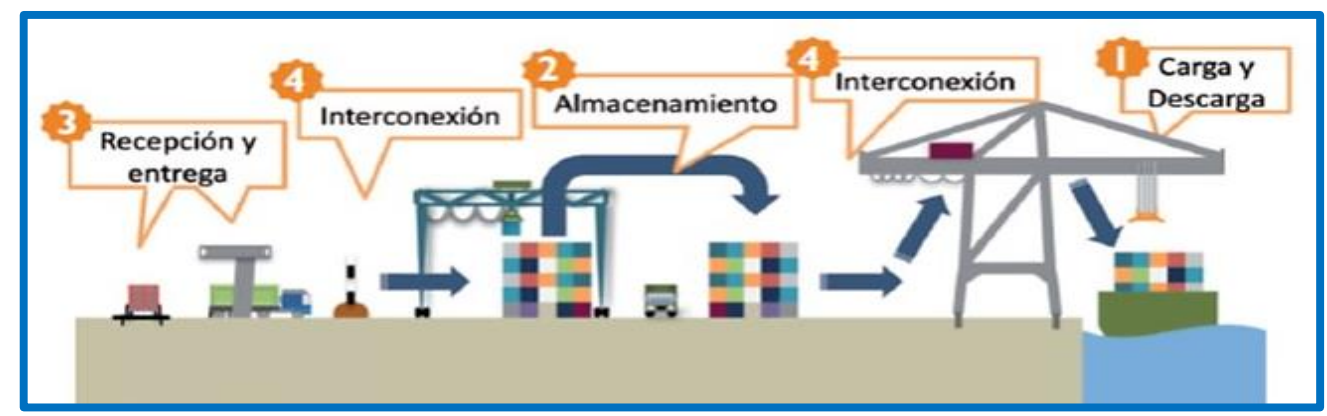

This work is licensed under a Creative Commons Attribution-NonCommercial-NoDerivatives 4.0 International License (CC BY-NC- 


\section{Fig. 3 - Subsistemas de una terminal portuaria de contenedores}

\subsection{La capacidad de una terminal portuaria de contenedores}

La capacidad de una terminal portuaria, en un periodo de tiempo (generalmente un año), se define como el máximo tráfico al que se puede dar servicio en un escenario definido. En función de la caracterización del referido escenario se alcanzan distintos conceptos de capacidad. Conviene subrayar que la capacidad de una terminal evoluciona con el tiempo en la medida que cambian las condiciones del escenario. En la Tabla 1 y Tabla 2 se resumen las categorías y definiciones del concepto de performance empleado en la presente ponencia.

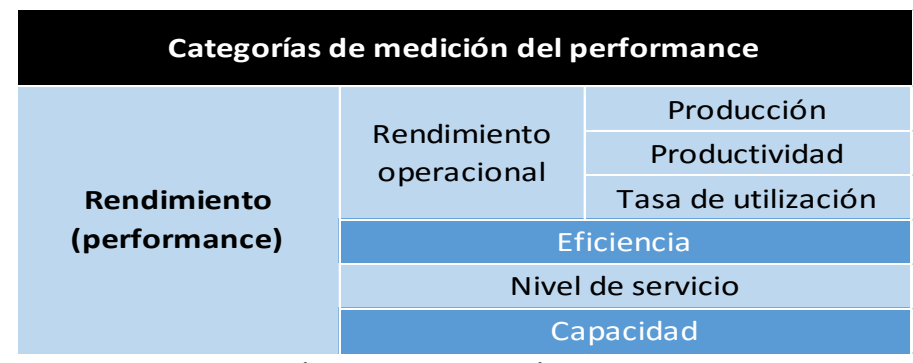

Tabla 1 - Esquema de las categorías de medición del rendimiento (performance)

\begin{tabular}{|c|l|}
$\begin{array}{c}\text { Categoría de } \\
\text { medición }\end{array}$ & \multicolumn{1}{c|}{ Definición } \\
\hline $\begin{array}{c}\text { Rendimiento } \\
\text { operacional }\end{array}$ & $\begin{array}{l}\text { Agrupación conceptual de las mediciones de producción } \\
\text { (tráfico), productividad y tasa de utilización }\end{array}$ \\
\hline Eficiencia & $\begin{array}{l}\text { Cociente de una suma ponderada de inputs por una suma } \\
\text { ponderada de outputs. Es la generalización del concepto de } \\
\text { productividad para varios recursos }\end{array}$ \\
\hline Nivel de servicio & $\begin{array}{l}\text { Expresa la calidad del servicio ofrecida a los clientes y usuarios } \\
\text { de la terminal }\end{array}$ \\
\hline Capacidad & $\begin{array}{l}\text { Producción o productividad de la terminal para un nivel de } \\
\text { servicio determinado }\end{array}$ \\
\hline
\end{tabular}

Tabla 2 - Categorías de medición del rendimiento (performance): definiciones

Desde la perspectiva de la terminal conformada por un conjunto de subsistemas, el consenso es generalizado: se define como capacidad de la terminal, en un periodo temporal y para un escenario (nivel de servicio), como la menor de las respectivas capacidades de los subsistemas que la integran: subsistema de carga y descarga de buques (línea de atraque), subsistema de interconexión, subsistema de almacenamiento y subsistema de recepción y entrega terrestre (Fig. 4). Es evidente que se trata de una visión analítica simplificada puesto que los subsistemas interaccionan de manera natural de modo que el planteamiento va a requerir la generación de una serie de hipótesis de trabajo que permitan el aislamiento de cada subsistema para el cálculo de su capacidad. 


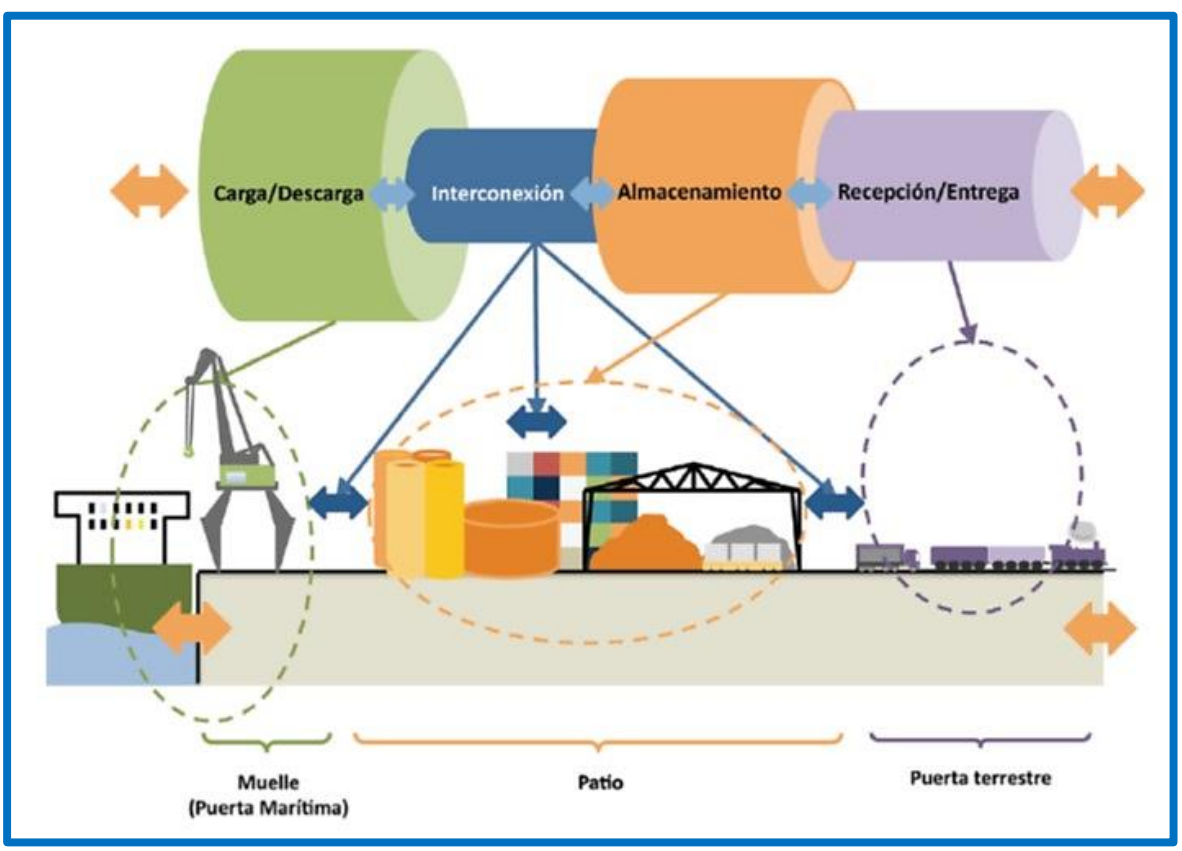

Fig. 4 - Capacidad de la terminal portuaria por subsistemas

\section{CAPACIDAD Y NIVEL DE SERVICIO POR LÍNEA DE ATRAQUE}

\subsection{Capacidad por línea de atraque de una terminal portuaria de contenedores}

La capacidad anual de la línea de atraque (subsistema de carga/descarga) de una terminal es igual al producto del número de puestos de atraque, por la tasa de ocupación de muelle, por las horas operativas anuales, y por la productividad horaria de los buques ponderada por el tiempo de atraque (Fig. 5):

Donde,

$$
C_{L A}=n \times \phi \times t_{a n ̃ o} \times P
$$

CLA: capacidad anual por línea de atraque (toneladas, contenedores o TEUs, unidades por año).

n: número de puestos de atraque.

$\boldsymbol{\phi}$ : Tasa de ocupación admisible. Es función del número de puestos de atraque, de la calidad de servicio asociada a la espera relativa $(\varepsilon)$ y de la caracterización de la distribución de llegadas de los buques y de los tiempos de servicio.

taño: Horas operativas de la terminal al año. Es función de los días que opera el puerto y de las condiciones laborales (turnos diarios, número de horas por turno, etc.) y meteorológicas.

P: Productividad horaria de los buques ponderada por el tiempo de atraque. Resulta del cociente entre el volumen anual de mercancías manipuladas y la suma de los tiempos brutos de atraque. Es función del número y prestaciones de los equipos empleados, la pericia de los manipuladores y la conexión con los otros subsistemas, entre otros factores.

Los métodos analíticos y de simulación contemplan la línea de atraque de la terminal como un sistema de esperas dotado de $\mathrm{n}$ puestos de servicio (en este caso serían $\mathrm{n}$ atraques): 
- con una función de distribución de probabilidades de llegadas de buques (A); y,

- otra función de distribución de probabilidades de tiempo de servicio (B).

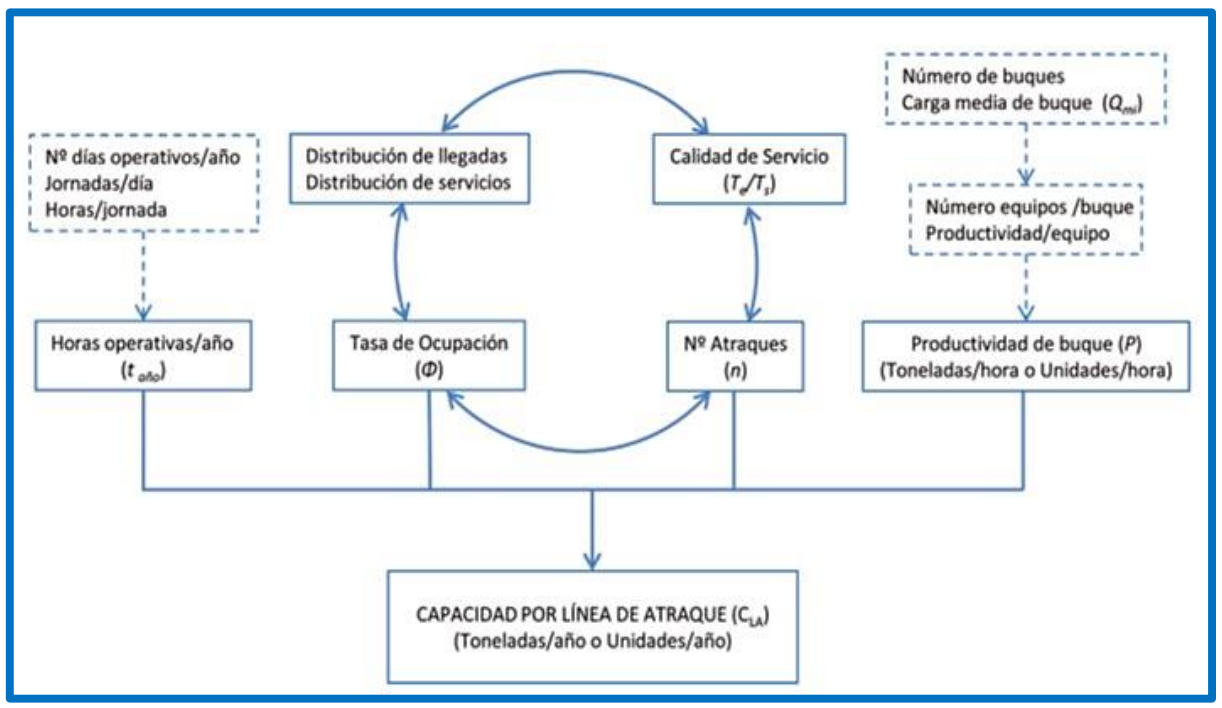

Fig. 5 - Cálculo de la capacidad por línea de atraque

El sistema de esperas queda definido por estas distribuciones (la de llegadas y la de tiempos de servicio) y el número de atraques: $\mathrm{A} / \mathrm{B} / \mathrm{n}$. Las funciones de distribución pueden ser exponenciales ( $\mathrm{M}$-también llamadas de Poisson, marcovianas o aleatorias-), Erlang de orden $\mathrm{K}\left(\mathrm{E}_{\mathrm{K}}\right)$, constantes $(\mathrm{D})$, hiperexponenciales $(\mathrm{H})$, u otra cualquiera $(\mathrm{G})$.

La capacidad anual por línea de atraque de una terminal no es directamente proporcional al número de atraques ya que a mayor número de atraques, para la misma calidad de servicio o espera relativa, crece la tasa de ocupación admisible. Por ejemplo, una terminal M/E4/3, para una calidad de servicio (espera relativa) de 0,1 es un $36 \%$ más productiva por atraque que una de 2 atraques. Así, la terminal de 2 atraques, para alcanzar la misma capacidad unitaria por metro de línea de atraque requiere operar a un $36 \%$ más de productividad de buque atracado: por ejemplo a 75 cont/h buque atracado frente a 55 (Fig. 6).

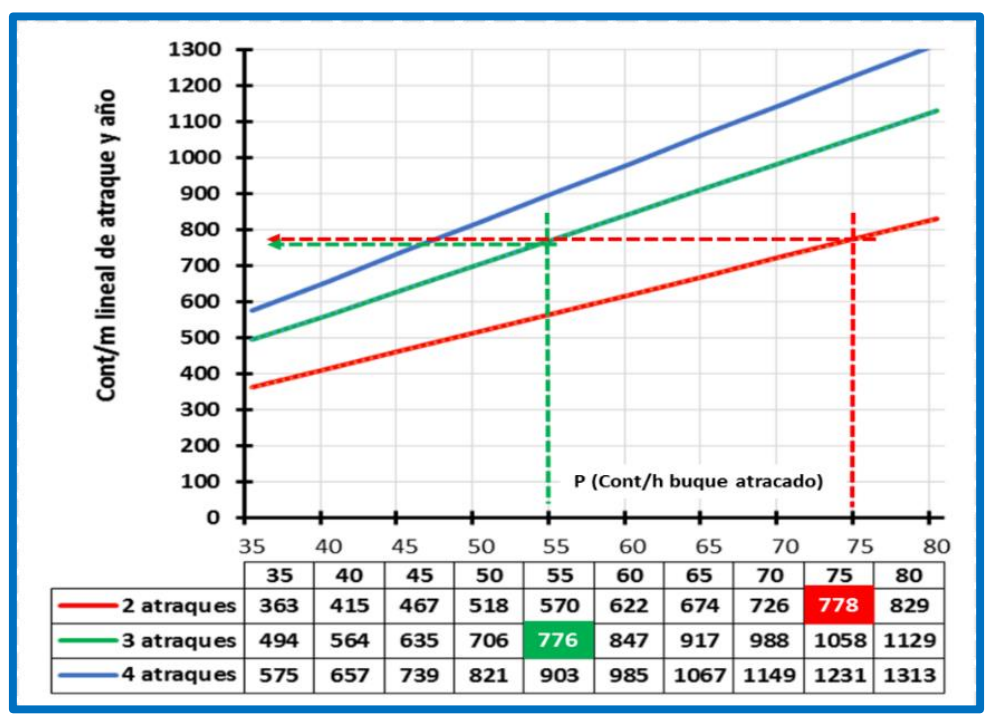

Fig. 6 - No linealidad de la capacidad en función del número de atraques: equivalencia en términos de productividad de buque atracado. Sistema $\mathrm{M} / \mathbf{E} 4 / 2$ y $\mathrm{M} / \mathbf{E} 4 / 3$ para $\varepsilon=0,1$ 


\subsection{Caracterización del nivel de servicio por línea de atraque}

El concepto de nivel de servicio (Monfort et al., 2011) ha sido desarrollado para proporcionar una medida de la calidad percibida por los clientes y usuarios del sistema. Por otro lado facilita la introducción de un escenario de condiciones límite que conlleva el cálculo de la capacidad portuaria. En el caso del subsistema de carga/descarga, el nivel de servicio ofrecido a la naviera (medida de la satisfacción del cliente) es función de dos indicadores fundamentales (Fig. 7): por un lado, la productividad del buque atracado P, y, por otro, la espera relativa $(\varepsilon)$ que expresa el ratio entre el tiempo de espera o fondeo y el tiempo de servicio del buque.

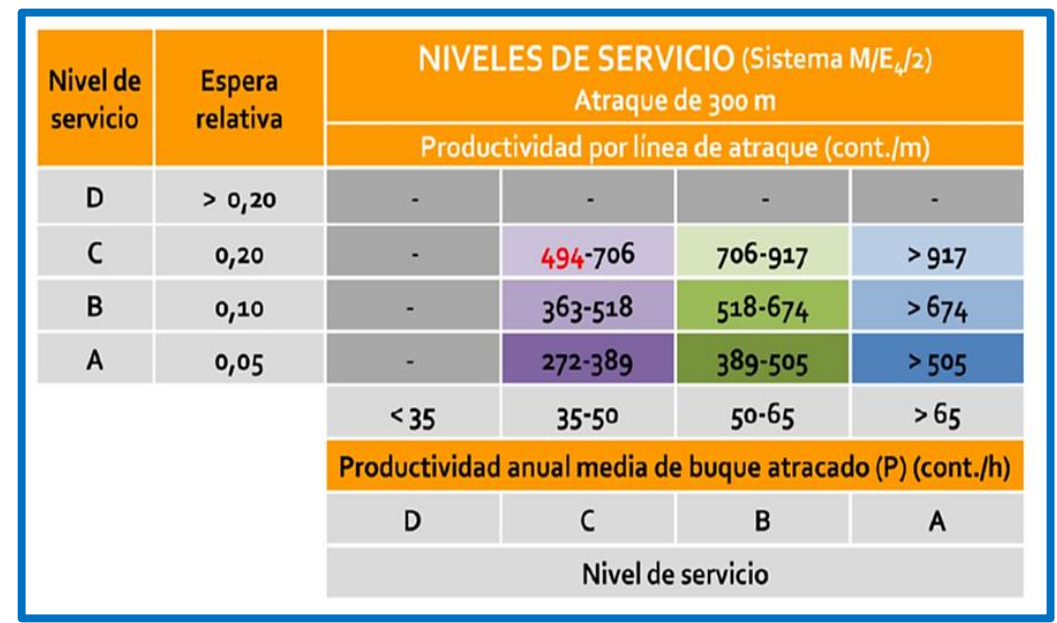

\section{Fig. 7 - Cálculo de la capacidad por línea de atraque}

Por otro lado, definido el nivel de servicio en los términos indicados para el correspondiente sistema de llegadas, servicios y número de atraques representativo de la terminal se obtiene el valor del indicador de capacidad por línea de atraque (cont/m lineal de atraque y año) como se muestra en la Figura 8.

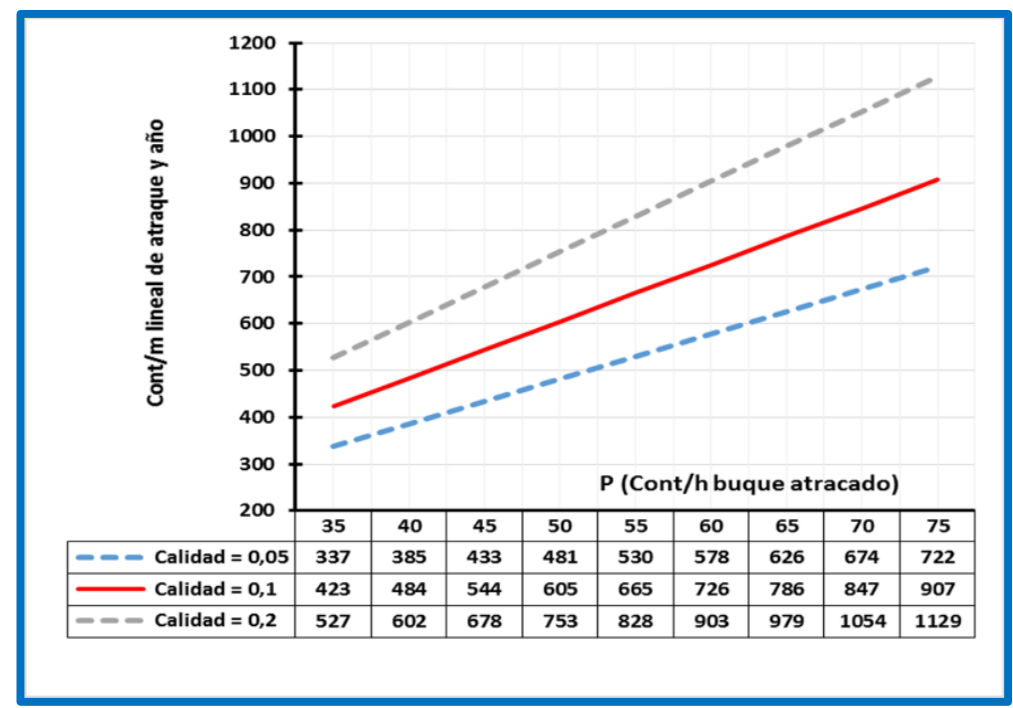

Fig. 8 - Capacidad por línea de atraque para diversos niveles de servicio (Caso M/E4/3 con atraques de $350 \mathrm{~m}$ ) 
Diversos autores y recomendaciones (Agerschou, 2004; Monfort et al., 2011; ROM 2.0 $2011,2012)$ acotan el valor referencial de la calidad de servicio o espera relativa ( $\varepsilon$ ) de los buques en terminales de contenedores entre 0,1 y 0,2 , en función del equilibrio de la oferta/demanda del tráfico contenedorizado. Del mismo modo, el valor de la productividad de atraque (P) debe adecuarse a la demanda (mercado) en función de la oferta de las TCs alternativas, contemplando para ello el tamaño medio de las escalas y el indicador de movimientos o contenedores por metro de eslora, el número de movimientos anuales por grúa de muelle y la distancia entre grúas. Por ejemplo, con 3 grúas por atraque de $300 \mathrm{~m}$ (100 m de distancia entre grúas) son perfectamente alcanzables productividades de atraque (brutas) de $60 \mathrm{mov} / \mathrm{h}$ que resultan en capacidades por metro de línea de atraque más o menos elevadas en función del número de atraques como se ha justificado a lo largo de la ponencia.

\section{CONCLUSIONES}

El contrato de concesión de una terminal de contenedores debe incorporar en la cláusula del correspondiente al nivel de servicio a ofertar en la terminal, un apartado relativo al nivel de servicio por línea de atraque con indicación expresa de la metas de calidad de servicio (espera relativa de los buques) y de productividad de buque atracado (P).

Por otro lado, es imprescindible que tanto la definición como la fórmula de cálculo del indicador no admita interpretaciones; y se asegure la disponibilidad de los datos necesarios para su determinación. Este es un aspecto crítico porque las discusiones a posteriori entre la autoridad portuaria y el operador son interminables.

Los referidos indicadores debieran contemplarse como uno de los elementos a ponderar como criterio de adjudicación de una terminal pública de contenedores.

\section{REFERENCIAS}

AGERSCHOU, H. (2004). Facilities requirements. Planning and design of ports and marine terminals, chapter 1 pp. 5-20. Thomas Telford Ltd, London.

BROOKS, M. R.; CULLINANE, K. (2006). Introduction: Devolution, Port Governance and Port Performance". Research in Transportation Economics, 17 (1), pp 3-28.

COMISIÓN TÉCNICA DE LA ROM 2.0. ROM 2.0-2011 (2012). Recomendaciones para el proyecto y ejecución de obras de atraque y amarre. Puertos del Estado, Madrid.

DONNET, B. (1910). Los puertos secundarios de Valencia. Revista de Obras Públicas, pp 572-574.

MONFORT, A.; AGUILAR, J.; GÓMEZ-FERRER, R.; et al. (2001). Terminales marítimas de contenedores: el desarrollo de la automatización. Fundación Valenciaport, Valencia.

MONFORT, A.; AGUILAR, J.; VIEIRA, P.; MONTERDE, N.; OBRER, R.; CALDUCH, D.; MARTÍN, A. M.; SAPIÑA, R. (2011). Manual de Capacidad Portuaria: Aplicación a Terminales de Contenedores. Fundación Valenciaport, Valencia.

RODRÍGUEZ, F. (1977). Capacidad de los muelles. MOPT, Madrid. 